TAIWANESE JOURNAL OF MATHEMATICS

Vol. 3, No. 3, pp. 311-322, September 1999

\title{
$\epsilon$-OPTIMALITY AND DUALITY FOR FRACTIONAL PROGRAMMING
}

\author{
Jen-Chwan Liu* and Kazunori Yokoyama
}

\begin{abstract}
We use the parametric approach and exact penalty function to establish the Karush-Kuhn-Tucker type necessary and sufficient conditions for an $\epsilon$-optimum of nondifferentiable fractional objective function subject to nondifferentiable convex inequality constraint, linear equality constraints and abstract constraints. Subsequently, these optimality criteria are utilized as a basis for constructing one dual problem, and duality theorems are presented.
\end{abstract}

\section{INTRODUCTION}

Several authors have been interested recently in $\epsilon$-approximate solutions for nonlinear programming. For details, one can consult [5,7-12, 14-17]. In particular, Strodiot, Nguyen, and Heukemes [14] derived $\epsilon$-optimality conditions of the Karush-Kuhn-Tucker type for points which are within $\epsilon$ of being optimal to the problem of minimizing a nondifferentiable convex objective function subject to nondifferentiable convex inequality constraint, linear equality constraints and abstract constraints, and they showed how it is possible to construct a bundle algorithm based on the $\epsilon$-optimality conditions; that is, $\epsilon$-optimality conditions may offer clues for convergence analysis of algorithms. In [12], Loridan derived some properties of $\epsilon$-efficient point solutions for vector minimization problems and used the Ekeland's variational principle [2] to establish the $\epsilon$-Pareto optimality and $\epsilon$-quasi Pareto optimality. In [8], Liu also adapted the same approach to obtain the $\epsilon$-duality theorem of nondifferentiable nonconvex multiobjective programming.

Received November 25, 1997; revised March 17, 1998.

Communicated by S.-Y. Shaw.

1991 Mathematics Subject Classification: 26A51, 49N15, 90C32.

Key words and phrases: Fractional programs, parametric approach, penalty functions, $\epsilon$ optimality.

* The author is partially supported by NSC, Taiwan. 
Recently, several authors $[1,3,4,9,11,13,15,16,18]$ have used an exact penalty function to transform the nonlinear scalar programming problem into an unconstrained problem and derived the $\epsilon$-optimality. In [16], Yokoyama was concerned with the $\epsilon$-approximate solutions and extended some results of [15] to the vector minimization problems. Yokoyama transformed the vector problems into the unconstrained problems by using the exact penalty functions and showed the $\epsilon$-optimality criteria by estimating the penalty parameter in terms of $\epsilon$-approximate solutions for the associated dual problems. In [9], Liu also used the exact penalty function to transform the inequality multiobjective programming problem into a scalar unconstrained problem and to derive the Karush-Kuhn-Tucker type conditions in which Lagrange multipliers of objective functions are one, and he employed the optimality of the multiobjective programming to construct the Wolfe-type dual problem and derived duality theorems.

Ibaraki [6] used a parametric approach to solve fractional programming problems. Therefore, we hope to use the same approach to derive $\epsilon$-optimality for fractional programming. We organize this paper as follows. Some definitions and notations are given in Section 2. In Section 3, we use a parametric approach to derive the Karush-Kuhn-Tucker type necessary and sufficient conditions for $\epsilon$-optimality of fractional programming. When the optimality conditions are utilized, one parametric dual problem may be formulated and duality theorems are presented in Section 4. In Section 5, we use scalar penalty functions to establish the necessary and sufficient conditions of $\epsilon$ solution.

\section{Preliminaries}

Throughout the paper, let $\mathbb{R}^{n}$ be the $n$-dimensional Euclidean space and $\mathbb{R}_{+}^{n}$ be its non-negative orthant. Let $F$ be a nonempty subset of $\mathbb{R}^{n}$ defined by

$$
F=\left\{x \in \mathbb{R}^{n} \mid h_{i}(x) \leq 0 \text { for } 1 \leq i \leq p, A x=b, \text { and } x \in Q\right\},
$$

where $h_{i}$ are convex real-valued functions defined on $\mathbb{R}^{n}$ for $1 \leq i \leq p, A$ is an $m \times n$ matrix of rank $m, b$ is an $m$ vector, and $Q$ is a nonempty closed convex subset of $\mathbb{R}^{n}$.

We consider the following fractional programming problem:

$$
\begin{array}{lll}
(P) & \text { Minimize } & \frac{f(x)}{g(x)} \\
\text { subject to } & x \in F,
\end{array}
$$

where $f$ and $-g$ are convex real-valued functions defined on $\mathbb{R}^{n}$ and satisfying $g(x)>0$ and $f(x) \geq 0$ for all $x \in F$. Let $\epsilon$ be an element of $\mathbb{R}_{+}$and $\bar{x}$ be 
a feasible point for $(\mathrm{P})$; that is, $\bar{x} \in F$. The point $\bar{x}$ is called an $\epsilon$-optimal solution of $(\mathrm{P})$ if

$$
\frac{f(\bar{x})}{g(\bar{x})} \leq \frac{f(x)}{g(x)}+\epsilon, \quad \text { for all } x \in F .
$$

In order to simplify the complication of problem $(\mathrm{P})$, we consider the following nonfractional problem with a parameter $v \in \mathbb{R}$ :

$$
\begin{array}{lll}
\left(P_{v}\right) \quad \text { Minimize } & f(x)-v g(x) \\
& \text { subject to } \quad x \in F .
\end{array}
$$

If $f$ and $-g$ are convex and $v \geq 0$, then $f(x)-v g(x)$ is convex; whereas $f(x) / g(x)$ is not. Thus in many cases, $\left(P_{v}\right)$ is easier than $(\mathrm{P})$. Therefore, we hope to employ the $\epsilon$-optimality of $\left(P_{v}\right)$ to derive the $\epsilon$-optimality of $(\mathrm{P})$. Unfortunately, the result is not so obvious and direct, and partial revisions must be made. We shall need the following revised lemma; the proof is simple and so omitted.

Lemma 2.1. Let $\bar{x} \in F$ and $\bar{v}=f(\bar{x}) / g(\bar{x})-\epsilon$. Then $\bar{x}$ is an $\epsilon$-optimal solution of $(P)$ if and only if $\bar{x}$ is a $g(\bar{x}) \epsilon$-optimal solution of $\left(P_{\bar{v}}\right)$.

We also need the following definitions:

Definition 2.1 [14]. Let $h: \mathbb{R}^{n} \longrightarrow \mathbb{R} \cup\{+\infty\}$ be a convex function, finite at $\bar{x}$. The $\epsilon$-subdifferential of $h$ at $\bar{x}$ is the set $\partial_{\epsilon} h(\bar{x})$ defined by

$$
\partial_{\epsilon} h(\bar{x})=\left\{x^{*} \in \mathbb{R}^{n} \mid h(y) \geq h(\bar{x})-\epsilon+\left\langle x^{*}, y-\bar{x}\right\rangle \quad \text { for any } y \in \mathbb{R}^{n}\right\} .
$$

Definition 2.2 [14]. Let $C$ be a nonempty closed convex subset of $\mathbb{R}^{n}$. The $\epsilon$-normal cone of $C$ at $\bar{x}$ is the set $N_{\epsilon}(C ; \bar{x})$ defined by

$$
N_{\epsilon}(C ; \bar{x})=\left\{x^{*} \in \mathbb{R}^{n} \mid\left\langle x^{*}, y-\bar{x}\right\rangle \leq \epsilon \quad \text { for any } y \in C\right\} .
$$

In order to establish some necessary and sufficient conditions for a feasible point $\bar{x}$ to be an $\epsilon$-optimal solution for $(\mathrm{P})$, we give the following non-fractional programming problem considered by Strodiot, Nguyen and Heukemes [14]:

$$
\begin{array}{ll}
\left(P_{1}\right) \quad \text { Minimize } & S(x) \\
& \text { subject to } \quad x \in F,
\end{array}
$$

where $S$ is a convex continuous real-valued function defined on $\mathbb{R}^{n}$. 
Lemma 2.2 [14, Theorem 2.4]. Let $\epsilon \geq 0$, and let $\bar{x}$ be a feasible point for $\left(P_{1}\right)$. Suppose that the following constraint qualification of the Slater type holds true:

There exists an $x_{0} \in \mathbb{R}^{n}$ such that $h_{i}\left(x_{0}\right)<0$
for $i=1, \cdots, p, A x_{0}=b$, and $x_{0} \in \operatorname{int} Q$.

Then $\bar{x}$ is an $\epsilon$-optimal solution to $(P)$ if and only if there exist scalars $\overline{\epsilon_{i}} \geq 0$ for $i=0,1, \cdots, p, \overline{\epsilon_{q}} \geq 0$ and $\lambda_{i} \geq 0$ for $i=1, \cdots, p$, and a vector $\mu \in \mathbb{R}^{m}$ such that

$$
\begin{gathered}
0 \in \partial_{\overline{\epsilon_{0}}} S(\bar{x})+\sum_{i=1}^{p} \partial_{\overline{\epsilon_{i}}}\left(\lambda_{i} h_{i}\right)(\bar{x})+A^{\top} \mu+N_{\overline{\epsilon_{q}}}(Q ; \bar{x}), \\
\sum_{i=0}^{p} \overline{\epsilon_{i}}+\overline{\epsilon_{q}}-\epsilon \leq \sum_{i=1}^{p} \lambda_{i} h_{i}(\bar{x}) \leq 0 .
\end{gathered}
$$

\section{Necessary and Sufficient Conditions}

In this section, we use Lemmas 2.1 and 2.2 to derive a criterion of KarushKuhn-Tucker type which is necessary and sufficient for a feasible point $\bar{x}$ to be an $\epsilon$-optimal solution for $(\mathrm{P})$.

Theorem 3.1. Let $\bar{x} \in F$ and $0 \leq \epsilon \leq f(\bar{x}) / g(\bar{x})$. Suppose that the constraint qualification $(C Q)$ as defined in Lemma 2.2 is satisfied at $\bar{x}$. Then $\bar{x}$ is an $\epsilon$-optimal solution to $(P)$ if and only if there exist scalars $\overline{\epsilon_{0 j}} \geq 0, j=$ $1,2, \overline{\epsilon_{i}} \geq 0, i=1,2, \cdots, p, \overline{\epsilon_{q}} \geq 0$ and $\overline{\lambda_{i}} \geq 0, i=1, \cdots, p$, and a vector $\mu \in \mathbb{R}^{m}$, and $\bar{v} \in \mathbb{R}_{+}$such that

$$
\begin{gathered}
0 \in \partial_{\overline{\epsilon_{01}}} f(\bar{x})+\partial_{\overline{\epsilon_{02}}}(\bar{v}(-g))(\bar{x})+\sum_{i=1}^{p} \partial_{\overline{\epsilon_{i}}}\left(\overline{\lambda_{i}} h_{i}\right)(\bar{x})+A^{\top} \mu+N_{\overline{\epsilon_{q}}}(Q ; \bar{x}), \\
f(\bar{x})-\bar{v} g(\bar{x})=\epsilon g(\bar{x}), \\
\overline{\epsilon_{01}}+\overline{\epsilon_{02}}+\sum_{i=1}^{p} \overline{\epsilon_{i}}+\overline{\epsilon_{q}}-\epsilon g(\bar{x}) \leq \sum_{i=1}^{p} \overline{\lambda_{i}} h_{i}(\bar{x}) \leq 0 .
\end{gathered}
$$

Proof. Suppose that $\bar{x}$ is an $\epsilon$-optimal solution of $(\mathrm{P})$. Let $\bar{v}=f(\bar{x}) / g(\bar{x})-$ $\epsilon \in \mathbb{R}_{+}$. By Lemma 2.1, $\bar{x}$ is a $g(\bar{x}) \epsilon$-optimal solution of $\left(P_{\bar{v}}\right)$. Thus, by Lemma 
2.2 , there exist scalars $\overline{\epsilon_{i}} \geq 0, i=0,1, \cdots, p, \overline{\epsilon_{q}} \geq 0$ and $\overline{\lambda_{i}} \geq 0, i=1, \cdots, p$, and a vector $\mu \in \mathbb{R}^{m}$ such that

$$
\begin{aligned}
& 0 \in \partial_{\bar{\epsilon}_{0}}(f+\bar{v}(-g))(\bar{x})+\sum_{i=1}^{p} \partial_{\overline{\epsilon_{i}}}\left(\overline{\lambda_{i}} h_{i}\right)(\bar{x})+A^{\top} \mu+N_{\overline{\epsilon_{q}}}(Q ; \bar{x}), \\
& \sum_{i=0}^{p} \overline{\epsilon_{i}}+\overline{\epsilon_{q}}-\epsilon g(\bar{x}) \leq \sum_{i=1}^{p} \overline{\lambda_{i}} h_{i}(\bar{x}) \leq 0 .
\end{aligned}
$$

Applying [5, Theorem 2.1], we have

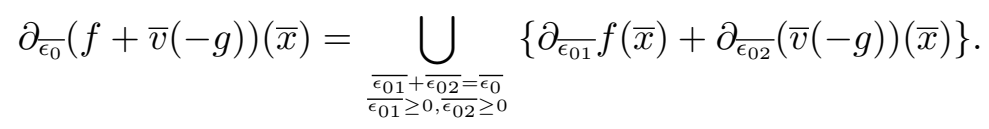

Thus, we have conditions (3.1), (3.2) and (3.3).

Conversely, if there exist scalars $\overline{\epsilon_{0 j}} \geq 0, j=1,2, \overline{\epsilon_{i}} \geq 0, i=1,2, \cdots, p$, $\overline{\epsilon_{q}} \geq 0$ and $\overline{\lambda_{i}} \geq 0, i=1, \cdots, p$, and a vector $\mu \in \mathbb{R}^{m}$, and $\bar{v} \in \mathbb{R}_{+}$such that (3.1), (3.2) and (3.3), then from [5, Theorem 2.1], we have

$$
\begin{aligned}
0 & \in \partial_{\overline{\epsilon_{01}}} f(\bar{x})+\partial_{\overline{\epsilon_{02}}}(\bar{v}(-g))(\bar{x})+\sum_{i=1}^{p} \partial_{\bar{\epsilon}_{i}}\left(\overline{\lambda_{i}} h_{i}\right)(\bar{x})+A^{\top} \mu+N_{\overline{\epsilon_{q}}}(Q ; \bar{x}) \\
& \subset \bigcup_{\substack{\bar{\epsilon}_{01}+\overline{\epsilon_{02}}=\overline{\epsilon_{0}} \\
\bar{\epsilon}_{01} \geq 0, \overline{\bar{\epsilon}_{02}} \geq 0}}\left\{\partial_{\overline{\overline{0}_{01}}} f(\bar{x})+\partial_{\overline{\epsilon_{02}}}(\bar{v}(-g))(\bar{x})\right\}+\sum_{i=1}^{p} \partial_{\overline{\bar{\epsilon}_{i}}}\left(\overline{\lambda_{i}} h_{i}\right)(\bar{x})+A^{\top} \mu+N_{\overline{\epsilon_{q}}}(Q ; \bar{x}) \\
& =\partial_{\overline{\epsilon_{0}}}(f+\bar{v}(-g))(\bar{x})+\sum_{i=1}^{p} \partial_{\overline{\epsilon_{i}}}\left(\overline{\lambda_{i}} h_{i}\right)(\bar{x})+A^{\top} \mu+N_{\overline{\epsilon_{q}}}(Q ; \bar{x})
\end{aligned}
$$

It follows from Lemma 2.2 that $\bar{x}$ is a $g(\bar{x}) \epsilon$-optimal solution of $\left(P_{\bar{v}}\right)$. Therefore, by Lemma 2.1, $\bar{x}$ is an $\epsilon$-optimal solution of $(\mathrm{P})$. Thus, the proof is complete.

\section{4. $\epsilon$-Duality Theorem}

From the $\epsilon$-optimality conditions for the problem $(\mathrm{P})$ in the preceding section, we can formulate the following parametric dual problem:

(D) Maximize $v$ subject to

$0 \in \partial_{\epsilon_{01}} f(u)+\partial_{\epsilon_{02}}(v(-g))(u)+\sum_{i=1}^{p} \partial_{\epsilon_{i}}\left(\lambda_{i} h_{i}\right)(u)+A^{\top} \mu+N_{\epsilon_{q}}(Q ; u)$, 


$$
\begin{aligned}
& f(u)-v g(u) \geq \epsilon g(u), \\
& \epsilon_{01}+\epsilon_{02}+\sum_{i=1}^{p} \epsilon_{i}+\epsilon_{q}-\epsilon g(u) \leq \sum_{i=1}^{p} \lambda_{i} h_{i}(u) \leq 0, \\
& A^{\top} u=b, \\
& \epsilon_{0 i} \geq 0 \text { for } i=1,2, \epsilon_{i} \geq 0 \text { for } 1 \leq i \leq p, \epsilon_{q} \geq 0, \\
& (u, v, \lambda, \mu) \in \mathbb{R}^{n} \times \mathbb{R}_{+} \times \mathbb{R}_{+}^{p} \times \mathbb{R}^{m} .
\end{aligned}
$$

We denote by $F_{D}$ the set of all feasible solutions $\left(u, v, \lambda, \mu, \epsilon_{01}, \epsilon_{02}, \epsilon_{1}, \epsilon_{2}, \cdots, \epsilon_{p}\right.$, $\left.\epsilon_{q}\right)$ of problem (D). The point $\left(\bar{u}, \bar{v}, \bar{\lambda}, \bar{\mu}, \overline{\epsilon_{01}}, \overline{\epsilon_{02}}, \overline{\epsilon_{1}}, \overline{\epsilon_{2}}, \cdots, \overline{\epsilon_{p}}, \overline{\epsilon_{q}}\right) \in F_{D}$ is called an $\epsilon$-optimal solution of (D) if $\bar{v} \geq v-\epsilon$ for all $\left(u, v, \lambda, \mu, \epsilon_{01}, \epsilon_{02}, \epsilon_{1}, \epsilon_{2}, \cdots, \epsilon_{p}, \epsilon_{q}\right)$ $\in F_{D}$.

Theorem 4.1 (Duality Theorem). Let $\bar{x} \in F$ and $0 \leq \epsilon \leq f(\bar{x}) / g(\bar{x})$. Suppose that the constraint qualification $(C Q)$ as defined in Lemma 2.2 is satisfied at $\bar{x}$. If $\bar{x}$ is an $\epsilon$-optimal solution of $(P)$, then there exist scalars $\overline{\epsilon_{0 j}} \geq 0, j=1,2, \overline{\epsilon_{i}} \geq 0, i=1,2, \cdots, p, \overline{\epsilon_{q}} \geq 0$ and $\overline{\lambda_{i}} \geq 0, i=1, \cdots, p$, and $a$ vector $\bar{\mu} \in \mathbb{R}^{m}$, and $\bar{v} \in \mathbb{R}_{+}$such that $\left(\bar{x}, \bar{v}, \bar{\lambda}, \bar{\mu}, \overline{\epsilon_{01}}, \overline{\epsilon_{02}}, \overline{\epsilon_{1}}, \overline{\epsilon_{2}}, \cdots, \overline{\epsilon_{p}}, \overline{\epsilon_{q}}\right)$ is an $\epsilon$-optimal solution of $(D)$.

Proof. With Theorem 3.1, we conclude that $\left(\bar{x}, \bar{v}, \bar{\lambda}, \bar{\mu}, \overline{\epsilon_{01}}, \overline{\epsilon_{02}}, \overline{\epsilon_{1}}, \overline{\epsilon_{2}}, \cdots, \overline{\epsilon_{p}}\right.$, $\left.\overline{\epsilon_{q}}\right)$ is a feasible solution of (D) and

$$
\bar{v}=\frac{f(\bar{x})}{g(\bar{x})}-\epsilon .
$$

Let $K(u)=A u-b$. For any feasible solution $\left(u, v, \lambda, \mu, \epsilon_{01}, \epsilon_{02}, \epsilon_{1}, \epsilon_{2}, \cdots, \epsilon_{p}, \epsilon_{q}\right)$ $\in F_{D}$, by (4.1), there exist $x_{1}^{*} \in \partial_{\epsilon_{01}} f(u), x_{2}^{*} \in \partial_{\epsilon_{02}}(-v g)(u), y_{i}^{*} \in \partial_{\epsilon_{i}}\left(\lambda_{i} h_{i}\right)(u)$ for $1 \leq i \leq p$ and $z^{*} \in N_{\epsilon_{q}}(Q ; u)$ such that

$$
x_{1}^{*}+x_{2}^{*}+\sum_{i=1}^{p} y_{i}^{*}+\nabla K^{\top}(u) \mu+z^{*}=0 .
$$

Using the characterization of the $\epsilon$-subgradient, we obtain

$$
\begin{aligned}
f(\bar{x})-f(u) & \geq\left\langle x_{1}^{*}, \bar{x}-u\right\rangle-\epsilon_{01}, \\
-v g(\bar{x})+v g(u) & \geq\left\langle x_{2}^{*}, \bar{x}-u\right\rangle-\epsilon_{02}, \\
\lambda_{i} h_{i}(\bar{x})-\lambda_{i} h_{i}(u) & \geq\left\langle y_{i}^{*}, \bar{x}-u\right\rangle-\epsilon_{i}, \quad 1 \leq i \leq p, \\
0 & \geq\left\langle z^{*}, \bar{x}-u\right\rangle-\epsilon_{q},
\end{aligned}
$$


and

$$
\left.\nabla^{\top} K(u)(\bar{x}-u)=0 . \quad \text { (by the feasibility of } \bar{x} \text { and }(4.4)\right)
$$

From this and (4.8), (4.2), and (4.3), we have

$$
\begin{aligned}
& f(\bar{x})-v g(\bar{x})+\sum_{i=1}^{p} \lambda_{i} h_{i}(\bar{x}) \\
& \geq f(u)-v g(u)+\sum_{i=1}^{p} \lambda_{i} h_{i}(u)+\left\langle x_{1}^{*}+x_{2}^{*}+\sum_{i=1}^{p} y_{i}^{*}+z^{*}, \bar{x}-u\right\rangle \\
& -\left(\epsilon_{01}+\epsilon_{02}+\sum_{i=1}^{p} \epsilon_{i}+\epsilon_{q}\right) \geq \epsilon g(u)-\left(\epsilon_{01}+\epsilon_{02}+\sum_{i=1}^{p} \epsilon_{i}+\epsilon_{q}\right) \\
& +\sum_{i=1}^{p} \lambda_{i} h_{i}(u)+\left\langle-\nabla^{\top} K(u) \mu, \bar{x}-u\right\rangle \quad(\text { by }(4.2) \text { and }(4.8)) \\
& \geq 0 \quad(\text { by }(4.3)) .
\end{aligned}
$$

It follows that

$$
f(\bar{x})-v g(\bar{x})+\sum_{i=1}^{p} \lambda_{i} h_{i}(\bar{x}) \geq 0 .
$$

Using the feasibility of $\bar{x}$ and $\lambda \in \mathbb{R}_{+}^{p}$, we have

$$
\sum_{i=1}^{p} \lambda_{i} h_{i}(\bar{x}) \leq 0 .
$$

Consequently, (4.9) and (4.10) yield

$$
f(\bar{x})-v g(\bar{x}) \geq 0 .
$$

From (4.7) and (4.11), we have

$$
\bar{v}+\epsilon=\frac{f(\bar{x})}{g(\bar{x})} \geq v .
$$

Thus, the proof of the theorem is complete.

Theorem 4.2 (Converse Duality). Let $\bar{x}$ be a feasible solution of $(P)$. If $\left(\bar{x}, \bar{v}, \bar{\lambda}, \bar{\mu}, \overline{\epsilon_{01}}, \overline{\epsilon_{02}}, \overline{\epsilon_{1}}, \overline{\epsilon_{2}}, \cdots, \overline{\epsilon_{p}}, \overline{\epsilon_{q}}\right)$ is a feasible solution of $(D), \bar{x}$ is an $\epsilon$ optimal solution of $(P)$.

Proof. This follows from Theorem 3.1. 


\section{Exact Penalty Functions}

In this section, we employ penalty functions to derive Karush-Kuhn-Tucker type necessary and sufficient $\epsilon$-optimality conditions without constraint qualification for the following fractional programming:

$$
\begin{array}{lll}
(P 1) \quad \text { Minimize } & \frac{f(x)}{g(x)} \\
& \text { subject to } & h_{i}(x) \leq 0, \quad i=1,2, \cdots, p .
\end{array}
$$

We denote the feasible set $\left\{x \in \mathbb{R}^{n} \mid h_{i}(x) \leq 0,1 \leq i \leq p\right\}$ by $F_{1}$ and assume the feasible set $F_{1}$ is nonempty.

To transform the problem (P1) into an unconstrained problem, we use the exact penalty function introduced by Zangwill [18]

$$
\theta(x, \rho)=f(x)-\bar{v} g(x)+\rho \sum_{i=1}^{p} \max \left(0, h_{i}(x)\right),
$$

where $\rho>0$ and $\bar{v} \in \mathbb{R}$.

The associated unconstrained problem in which

$$
\left(\theta_{\rho}\right) \quad \text { Minimize } \theta(x, \rho)
$$

is called a penalized problem with respect to the penalty parameter $\rho$. Throughout this section, we let $0 \leq \epsilon \leq f(\bar{x}) / g(\bar{x})$ and $\bar{v}=f(\bar{x}) / g(\bar{x}))-\epsilon \geq 0$. The following necessary conditions for the $\epsilon$-optimality of (P1) can be derived:

Theorem 5.1. If there exists $\rho_{0}$ such that $\bar{x}$ is a $g(\bar{x}) \epsilon$-solution for $\left(\theta_{\rho}\right)$ for any $\rho \geq \rho_{0}$, then $\bar{x}$ is an $\epsilon$ - solution for $(P 1)$ and there exist scalars $\overline{\epsilon_{0 j}} \geq 0$ for $j=1,2, \overline{\epsilon_{i}} \geq 0$ for $i=1,2, \cdots, p$ and $\overline{\lambda_{i}} \geq 0$ for $i=1,2, \cdots, p$ such that

$$
\begin{aligned}
& 0 \in \partial_{\overline{\epsilon_{01}}} f(\bar{x})+\partial_{\overline{\epsilon_{02}}}(\bar{v}(-g))(\bar{x})+\sum_{i=1}^{p} \partial_{\overline{\bar{i}_{i}}}\left(\overline{\lambda_{i}} h_{i}\right)(\bar{x}), \\
& f(\bar{x})-\bar{v} g(\bar{x})=\epsilon g(\bar{x}), \\
& \overline{\epsilon_{01}}+\overline{\epsilon_{02}}+\sum_{i=1}^{p} \overline{\epsilon_{i}}-\epsilon g(\bar{x}) \leq \sum_{i=1}^{p} \overline{\lambda_{i}} h_{i}(\bar{x}) \leq 0 .
\end{aligned}
$$

Proof. If $\bar{x}$ is a $g(\bar{x}) \epsilon$-solution of problem $\left(\theta_{\rho}\right)$, then

$$
\theta(\bar{x}, \rho) \leq \theta(x, \rho)+g(\bar{x}) \epsilon \quad \text { for all } \quad x \in \mathbb{R}^{n} .
$$


If $\bar{x} \notin F_{1}$, then $\sum_{i=1}^{p} \max \left(0, h_{i}(\bar{x})\right)>0$.

From (5.2) and (5.4), we get:

for any $\rho \geq \rho_{0}>0$, and for all $x \in F_{1}, 0 \leq \rho \sum_{i=1}^{p} \max \left(0, h_{i}(\bar{x})\right) \leq f(x)-\bar{v} g(x)$.

Dividing by $\rho$ and letting $\rho \rightarrow+\infty$, we get $\sum_{i=1}^{p} \max \left(0, h_{i}(\bar{x})\right)=0$. This conclusion gives a contradiction and hence $\bar{x} \in F_{1}$.

Clearly,

$$
\theta(x, \rho)=f(x)-\bar{v} g(x) \quad \text { for all } \quad x \in F_{1} .
$$

From (5.4), we have

$$
f(\bar{x})-\bar{v} g(\bar{x}) \leq \theta(\bar{x}, \rho) \leq f(x)-\bar{v} g(x)+g(\bar{x}) \epsilon \quad \text { for all } \quad x \in F_{1} .
$$

Thus, $\bar{x}$ is a $g(\bar{x}) \epsilon$-optimal solution of $\left(P_{\bar{v}}\right)$. Therefore, by Lemma $2.1, \bar{x}$ is an $\epsilon$-optimal solution of (P1).

With (5.4) and the result of [5], we have

$$
0 \in \partial_{g(\bar{x}) \epsilon}\left(f(\cdot)+\bar{v}(-g(\cdot))+\rho \sum_{i=1}^{p} \max \left(0, h_{i}(\cdot)\right)\right)(\bar{x}) .
$$

Then, there exist scalars $\overline{\epsilon_{0 i}} \geq 0(i=1,2), \overline{\epsilon_{i}} \geq 0(1 \leq i \leq p), \alpha_{i} \geq 0(1 \leq i \leq$ $p)$, and $\eta_{i} \geq 0(1 \leq i \leq p)$ such that

$$
\begin{gathered}
\overline{\epsilon_{01}}+\overline{\epsilon_{02}}+\sum_{i=1}^{p} \overline{\epsilon_{i}}=g(\bar{x}) \epsilon \\
0 \in \partial_{\overline{\epsilon_{01}}} f(\bar{x})+\partial_{\overline{\epsilon_{02}}}(\bar{v}(-g(\bar{x})))+\sum_{i=1}^{p} \partial_{\eta_{i}}\left(\alpha_{i} \rho h_{i}\right)(\bar{x}),
\end{gathered}
$$

where$$
\alpha_{i} \leq 1,
$$$$
\eta_{i}+\max \left(0, h_{i}(\bar{x})\right)-\alpha_{i} \rho h_{i}(\bar{x})=\overline{\epsilon_{i}} \quad \text { for } \quad i=1, \ldots, p .
$$

By (5.5) and (5.7), we have

$$
\overline{\epsilon_{01}}+\overline{\epsilon_{02}}+\sum_{i=1}^{p} \eta_{i}-g(\bar{x}) \epsilon \leq \sum_{i=1}^{p} \alpha_{i} \rho h_{i}(\bar{x}) \leq 0 .
$$


Finally, we obtain the results (5.1) and (5.3) by setting

$$
\begin{array}{ll}
\overline{\lambda_{i}}=\alpha_{i} \rho, & 1 \leq i \leq p \\
\overline{\epsilon_{i}}=\eta_{i}, & 1 \leq i \leq p .
\end{array}
$$

Theorem 5.2. If there exist scalars $\overline{\epsilon_{0 j}} \geq 0$ for $j=1,2, \overline{\epsilon_{i}} \geq 0$ for $i=$ $1,2, \cdots, p$ and $\overline{\lambda_{i}} \geq 0$ for $i=1,2, \cdots, p$ such that the conditions $(5.1) \sim(5.3)$ hold, then $x_{0}$ is an $\epsilon$-optimal solution of (P1).

Proof. By (5.1), there exist $x_{1}^{*} \in \partial_{\overline{\epsilon_{01}}} f(\bar{x}), x_{2}^{*} \in \partial_{\overline{\epsilon_{02}}}(-\bar{v} g)(\bar{x})$, and $y_{i}^{*} \in$ $\partial_{\overline{\epsilon_{i}}}\left(\bar{\lambda}_{i} h_{i}\right)(\bar{x})$ for $1 \leq i \leq p$ such that

$$
x_{1}^{*}+x_{2}^{*}+\sum_{i=1}^{p} y_{i}^{*}=0 .
$$

Using the characterization of the $\epsilon$-subgradient, we obtain

$$
\begin{aligned}
f(x)-f(\bar{x}) & \geq\left\langle x_{1}^{*}, x-\bar{x}\right\rangle-\overline{\epsilon_{01}}, \\
-\bar{v} g(x)+\bar{v} g(\bar{x}) & \geq\left\langle x_{2}^{*}, x-\bar{x}\right\rangle-\overline{\epsilon_{02}}, \\
\overline{\lambda_{i}} h_{i}(x)-\overline{\lambda_{i}} h_{i}(\bar{x}) & \geq\left\langle y_{i}^{*}, x-\bar{x}\right\rangle-\overline{\epsilon_{i}}, \quad 1 \leq i \leq p .
\end{aligned}
$$

From this and (5.8), (5.3), we have

$$
\begin{aligned}
f(x) & -\bar{v} g(x)+\sum_{i=1}^{p} \overline{\lambda_{i}} h_{i}(x) \\
& \geq f(\bar{x})-\bar{v} g(\bar{x})+\sum_{i=1}^{p} \overline{\lambda_{i}} h_{i}(\bar{x})+\left\langle x_{1}^{*}+x_{2}^{*}+\sum_{i=1}^{p} y_{i}^{*}, x-\bar{x}\right\rangle \\
& -\left(\overline{\epsilon_{01}}+\overline{\epsilon_{02}}+\sum_{i=1}^{p} \overline{\epsilon_{i}}\right) \geq f(\bar{x})-\bar{v} g(\bar{x})-\epsilon g(\bar{x}) \quad \text { for all } \quad x \in F_{1} .
\end{aligned}
$$

Using the feasibility of $x$ and $\bar{\lambda} \in \mathbb{R}_{+}^{p}$, we have

$$
\sum_{i=1}^{p} \overline{\lambda_{i}} h_{i}(x) \leq 0
$$

Consequently, (5.9) and (5.10) yield

$$
f(x)-\bar{v} g(x) \geq f(\bar{x})-\bar{v} g(\bar{x})-\epsilon g(\bar{x}) \quad \text { for all } \quad x \in F_{1} .
$$

Thus, $\bar{x}$ is a $g(\bar{x}) \epsilon$-optimal solution of $\left(P_{\bar{v}}\right)$. Therefore, by Lemma $2.1, \bar{x}$ is an $\epsilon$-optimal solution of $(\mathrm{P} 1)$. Thus, the proof of the theorem is complete. 


\section{Some Remarks for Further Developments}

(1) The questions arise as to whether the results developed in this paper hold in multiobjective fractional programming problem (P2):

$$
\begin{array}{rll}
(P 2) \quad \text { Minimize } & \left(f_{1}(x) / g_{1}(x), \cdots, f_{r}(x) / g_{r}(x)\right) \\
\text { subject to } \quad & x \in F
\end{array}
$$

or in generalized fractional programming problem (P3):

$$
\begin{array}{ll} 
& \text { Minimize } \max _{1 \leq i \leq r} f_{i}(x) / g_{i}(x) \\
& \text { subject to } \quad x \in F .
\end{array}
$$

(2) Can the objective and constraint functions $f, g$ and $h$ in the fractional programming $(\mathrm{P})$ be replaced by other generalized convex functions such as pseudoconvex, quasiconvex, invex, preinvex, generalized $(F, \rho)$ convex, etc?

\section{ACKNOWLEDGMENTS}

The authors are thankful to Prof. Sen-Yen Shaw and the anonymous referee for their valuable comments and suggestions on an earlier version of the paper, especially on the proof of Theorem 5.1.

\section{REFERENCES}

1. D. P. Bertsekas, Necessary and sufficient conditions for a penalty method to be exact, Math. Programming 9 (1975), 87-99.

2. I. Ekeland, On the variational principle, J. Math. Anal. Appl. 47 (1974), 324-353.

3. S. P. Han and O. L. Mangasarian, Exact penalty functions in nonlinear programming, Math. Programming 17 (1979), 251-269.

4. S. P. Han and O. L. Mangasarian, A dual differentiable exact penalty function, Math. Programming 25 (1983), 293-306.

5. J. B. Hiriart-Urruty, $\epsilon$-Subdifferential calculus, in: Convex Analysis and Optimization, J. P. Aubin and R. B. Vinter, eds., Research Notes in Mathematics, Series No. 57, Pitman, Boston, MA, 1982, pp.43-92.

6. T. Ibaraki, Parametric approaches to fractional programming, Math. Programming 26 (1983), 345-362. 
7. S. S. Kutateladze, Convex $\epsilon$-programming, Soviet Math. Dokl. 20 (1979), 391393.

8. J. C. Liu, $\epsilon$-Duality theorem of nondifferentiable nonconvex multiobjective programming, J. Optim. Theory Appl. 69 (1991), 153-167.

9. J. C. Liu, $\epsilon$-Pareto optimality for nondifferentiable multiobjective programming via penalty function, J. Math. Anal. Appl. 198 (1996), 248-261.

10. P. Loridan, Necessary conditions for $\epsilon$-Optimalty, Math. Programming Study, 19 (1982), 140-152.

11. P. Loridan and J. Morgan, Penalty functions in $\epsilon$-programming and $\epsilon$-minimax problems, Math. Programming 26 (1983), 213-231.

12. P. Loridan, $\epsilon$-Solution in vector minimization problems, J. Optim. Theory Appl. 43 (1984), 265-267.

13. O. L. Mangasarian, Sufficiency of exact penalty minimization, SIAM J. Control Optim. 23 (1985), 30-37.

14. J. J. Strodiot, V. H. Nguyen and N. Heukemes, $\epsilon$-Optimal solutions in nondifferentiable convex programming and some related questions, Math. Programming 25 (1983), 307-328.

15. K. Yokoyama, $\epsilon$-Optimality criteria for convex programming problems via exact penalty functions, Math. Programming 56 (1992), 233-243.

16. K. Yokoyama, $\epsilon$-Optimality criteria for vector minimization problems via exact penalty functions, J. Math. Anal. Appl. 187 (1994), 296-305.

17. K. Yokoyama, Epsilon approximate solutions for multiobjective programming problems, J. Math. Anal. Appl. 203 (1996), 142-149.

18. W. I. Zangwill, Nonlinear programming via penalty functions, Management Sci. 13 (1967), 344-358.

Jen-Chwan Liu

Section of Mathematics, National Overseas Chinese Student University

Linkou 24499, PO Box 1-1337, Taiwan, R.O.C.

Kazunori Yokoyama

Department of Management and Information Sciences

Niigata University of Management

Kamo, Niigata, 959-13, Japan 